\title{
Use of monoclonal antibody E48 in diagnosing transitional cell carcinoma of urinary bladder
}

\author{
R Torenbeek, C E M Blomjous, J J Quak, S Ybema, C J L M Meijer
}

\begin{abstract}
Aims: To investigate the localisation of the $\mathbf{E 4 8}$ epitope and to determine the use of monoclonal antibody E48 for the identification of transitional cell carcinomas (TCC); and to determine if antigenic expression was affected by different standard fixation methods.

Methods: Biopsy specimens were labelled with $\mathrm{E} 48$ for immunoelectron microscopy. One hundred and nineteen tissue samples from 47 bladder carcinomas were tested for reactivity with $E 48$, using fresh frozen, sublimate formalin, and formalin fixed tissue. Thirteen undifferentiated bladder tumours and 10 undifferentiated prostatic carcinomas were incubated with E48 and prostate specific antigen.

Results: Reactivity to E48 was found in all grade 1 and 2 carcinomas and most $(83 \%)$ grade 3 tumours. At the ultrastructural level, expression was mainly associated with desmosomes and the cytoplasmic membrane. The reactivity of E48 was generally strong in fresh frozen tissue samples and remained preserved in fixed tissue samples. Ten of the 13 bladder carcinomas expressed E48; all prostatic tumours were totally negative.

Conclusions: E48 is a sensitive marker for transitional cell carcinoma and suitable for differentiation between urothelial and prostatic undifferentiated carcinoma. It can be used in routinely processed, formalin fixed, biopsy specimens.
\end{abstract}

For the past 10 years, immunohistochemical investigations of the antigenic determinants on urothelial bladder cancer cells have been increasingly used. ${ }^{1-5}$ Antigenic substances have been examined on tumour cells and in the plasma and urine samples of patients with urothelial neoplasms. ${ }^{6-9}$ Changes in antigenic expression in poorly differentiated carcinomas have been described for cytokeratins ${ }^{4510-13}$ and various blood group antigens, ${ }^{14-17}$ but biochemical markers that unequivocally predict the biological behaviour of bladder carcinoma have been energetically sought but without much success. There are therefore no reliable markers that specifically recognise benign and malignant urothelium for identifying the urothelial origin of poorly differentiated tumours. Such markers may be useful in tumours of uncertain origin, especially in metastases of unknown primaries.

Recently a new monoclonal antibody (E48) has been described which recognises a 22 kilodalton epitope that is exclusively expressed in squamous and transitional epithelium and their neoplastic derivatives. ${ }^{18}$ Staining of a large variety of normal human tissue specimens (141) showed that the E48 epitope was observed in the squamous epithelium of the skin, oropharynx, oesophagus, vagina and cervix, as well as the bladder urothelium, but not in simple epithelia or non-epithelial tissues. ${ }^{18}$ In a large series of neoplastic tissue specimens (276) labelling with E48 was almost exclusively found in squamous cell carcinoma and transitional cell carcinomas (TCC). ${ }^{18}$

In this study we investigated the ultrastructural localisation of the E48 epitope. To evaluate the use of E48 for diagnostic purposes we examined to what degree the expression in TCC is related to the grade of histological differentiation, and if the antigenic expression is affected by different standard fixation methods. Moreover, the diagnostic value was assessed to differentiate TCC from undifferentiated carcinomas in the trigone of the urinary bladder.

\section{Methods}

A detailed description of antigen preparation and absorption of antisera has been provided. ${ }^{18}$ Briefly, a surgically removed metastatic tumour of a moderately well differentiated squamous cell carcinoma from the larynx was homogenised and the single cells were used to immunise BALB/c mice. ${ }^{19}$ Dissociated spleen cells were fused with the nonproducing cell line SP-2/0, and antibodies were screened for reactivity with frozen sections from oral mucosa and the tumour used for immunisation. The selected monoclonal antibody was E48 (IgG1 class) and large amounts of antibody were obtained by mice producing ascites that had been primed by incomplete Freund's adjuvant.

For immunoelectron microscopy, a postembedding labelling method was recently developed in our laboratory. ${ }^{20}$ Biopsy specimens were immediately fixed in $2 \%$ paraformaldehyde in phosphate buffered saline (PBS), pH 7-7.4. After primary fixation the tissue specimens were washed three times with $0.1 \mathrm{M}$ maleate buffer and $3.5 \%$ sucrose (pH 6.5) and subsequently stained en bloc for two hours at $4^{\circ} \mathrm{C}$ with $2 \%$ uranyl acetate in the same buffer (pH 6.0). Tissue samples were
Correspondence to: Dr R Torenbeek Accepted for publication 2 October 1991 
Number of tissue samples showing diffuse or focal reactivity with $E 48$, related to the fixation method and grade of differentiation

\begin{tabular}{lccl}
\hline & \multicolumn{2}{c}{ Positive } & \\
\cline { 2 - 3 } TCC grade & Diffuse & Focal & Negative \\
\hline Fresh frozen $(n=42)$ & & & \\
$1(\mathrm{n}=9)$ & 9 & 0 & 0 \\
$2(\mathrm{n}=15)$ & 10 & 5 & 0 \\
$3(\mathrm{n}=18)$ & 10 & 5 & 3 \\
Sublimate formalin $(n=33)$ & & \\
$1(\mathrm{n}=6)$ & 5 & 1 & 0 \\
$2(\mathrm{n}=13)$ & 3 & 8 & 2 \\
$3(\mathrm{n}=14)$ & 4 & 6 & 4 \\
Formalin $(n=44)$ & & & \\
$1(\mathrm{n}=9)$ & 4 & 5 & 0 \\
$2(\mathrm{n}=17)$ & 4 & 10 & 3 \\
$3(\mathrm{n}=18)$ & 3 & 8 & 7 \\
\hline
\end{tabular}

then partly dehydrated in a series of graded alcohols (50-80\%), impregnated with LRWhite resin (Soft grade, BioRad Labs, Veenendaal, The Netherlands) and polymerised for 24 hours at $50^{\circ} \mathrm{C}$. Ultrathin sections (50$100 \mathrm{~nm}$ ) were collected on Formvar coated nickel grids (10 mesh) and incubated with E48 in $0 \cdot 1 \mathrm{M}$ TRIS-buffered saline (TBS) for two to four hours at room temperature. After several washes with TBS grids were incubated with colloidal gold $(10 \mathrm{~nm})$, conjugated to goat-anti-mouse immunoglobulins (GAMIgG-G10), Janssen Life Science Products, Olen, Belgium). Subsequently, grids were washed with TBS and distilled water, contrasted with Reynold's lead citrate and examined with a Zeiss EM 109.

To investigate the expression of $\mathrm{E} 48$ on TCCs 45 transureteral resected biopsy specimens and two cystectomy specimens were used of which various fixed tissue samples (fresh frozen 42), sublimate formalin fixation (33), and formalin fixation (44)) were available. In all, 119 specimens were screened for the presence of E48 antigen.

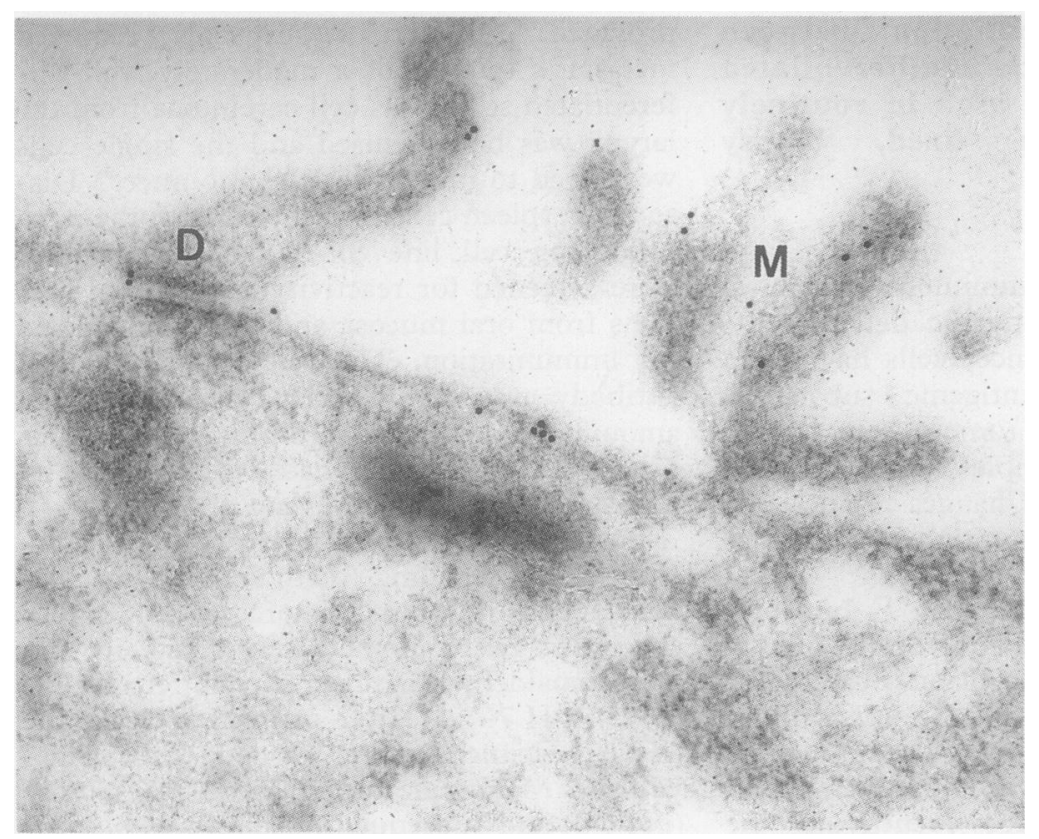

Figure 1 Immunoelectron microscopic analysis indicates that the E48 antigen is localised on desmosomes (D) and microvilli $(M)$ on the cytoplasmic membrane.
To investigate the value of E48 monoclonal antibody to differentiate between the urothelial or prostatic orgin of undifferentiated tumour in the trigone of the bladder, 13 undifferentiated (clinically confirmed) bladder tumours (formalin fixed) and 10 undifferentiated (prostate specific antigen (PSA) positive) prostatic carcinomas (formalin fixed and fresh frozen) were incubated with E48 and PSA.

Immediately after receiving the specimens samples were fixed in liquid nitrogen, phosphate buffered formalin (4\%), and sublimate formalin. For conventional histological examination sections were cut and stained with haematoxylin and eosin.

For immunoperoxidase staining fresh frozen tissue samples ( $4 \mu \mathrm{m}$ thick) were mounted on poly-L-lysine coated glass slides, air dried, and fixed in acetone for 10 minutes at room temperature. Sections also were cut from neutral buffered, formalin fixed, and sublimate fixed, paraffin wax embedded material. These sections were subsequently dewaxed, rehydrated, and treated with $0.5 \%$ $\mathrm{H}_{2} \mathrm{O}_{2}$ in methanol to block endogenous peroxidase activity. Sublimate formalin fixed slides were depigmented in Lugol's solution. For optimal staining results, sublimate formalin fixed samples were pretreated with tryp$\sin \left(0.5 \%\right.$ in $\left.\mathrm{CaCl}_{2} 0.5 \%\right)$ for 30 minutes at room temperature. Incubation time for E48 monoclonal antibody was one hour, followed by incubation (for 60 minutes) with a biotin labelled second layer horse-anti-mouse antibody (Vector Laboratories). After the latter the avidin-biotin-peroxidase complex was added. Sections were developed with diaminobenzidine for three minutes, counterstained with haematoxylin, and mounted with Depex.

\section{Results}

Immunoperoxidase labelling of the E48 antigen in a grade 2 TCC showed at ultrastructural level that E48 reactivity is largely associated with desmosomes and the cytoplasmic membrane. Furthermore, labelling was not seen on the basement membrane, nor on nuclear or cytoplasmic components (fig 1).

Forty seven bladder tumours were reexamined and classified (WHO system) as transitional cell carcinoma grade 1 (9), grade 2 (17), and grade 3 (18). The reactivity of E48 to TCC in relation to the histological grade and fixation method is shown in the table. Positive staining of the fresh frozen (42), sublimate formalin (33), and formalin fixed tumour samples (44) was found in $93 \%, 82 \%$, and $77 \%$ of the cases, respectively. Irrespective of the method of fixation $75 \%$ of the positive samples showed reactivity of the entire tumour sample (fig 2), whereas about $25 \%$ showed only focal staining. Positive staining of the grade 1,2 , and 3 TCCs varied slightly according to the fixation method used. Values for positive staining for grades 1 , 2 , and 3 carcinoma were, respectively, $100 \%$, $100 \%$, and $83 \%$ (fresh frozen), $100 \%, 85 \%$, and $71 \%$ (sublimate formalin fixed), and $100 \%$, $82 \%$, and $61 \%$ (formalin fixed). 


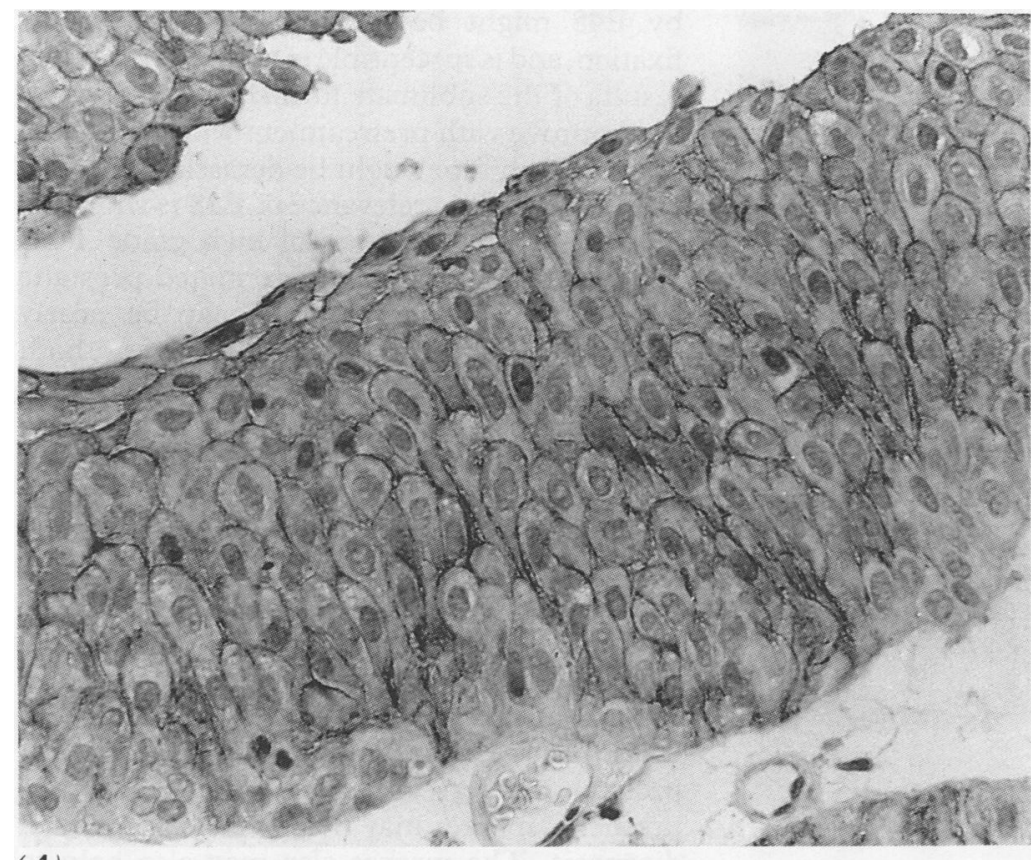

(A)

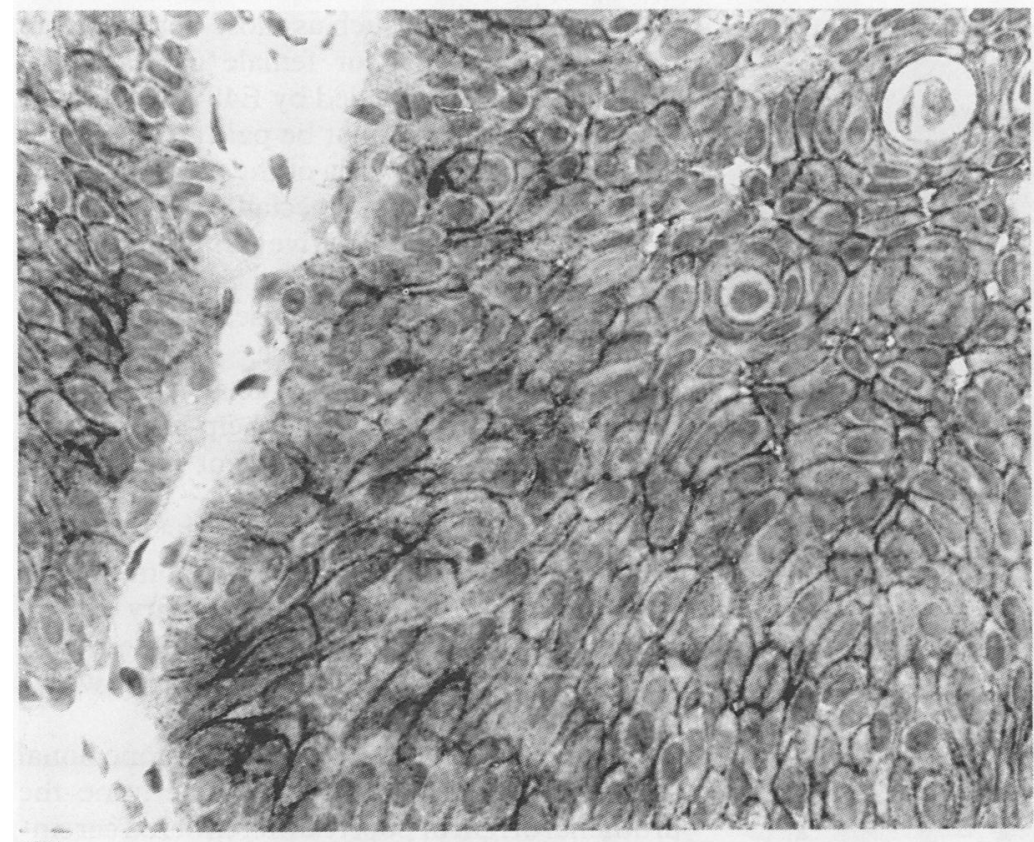

(B)

Figure 2 Papillary grade 1 (A) and solid grade 3 (B) transitional cell carcinoma showing cell membrane bound reactivity with $E 48$ in the entire tumour sample (formalin fixed). was absent in the unfixed slides and only very weak in the fixed slides.

To verify the specificity of E48, the staining results of 13 poorly differentiated TCCs of the bladder trigone were compared with 10 poorly differentiated adenocarcinomas of the prostate. Clinically there was no doubt about the urinary bladder or prostatic origin of these tumours. Ten of the 13 bladder carcinomas expressed the monoclonal antibody E48; all prostatic tumours (formalin fixed as well as unfixed tumour samples) were totally negative, except for squamous metaplastic foci in one of the samples (fig 3). Additional staining for PSA, on the other hand, showed a positive reaction in the prostatic carcinomas, in contrast to the bladder tumours.

Immunohistochemical staining of an incidental cervical lymph node metastasis of an unkown primary carcinoma showed reactivity to E48 (fig 4). Clinical evaluation of the patient resulted in the finding of a poorly differentiated solid TCC of the bladder with the same histological pattern as the lymph node metastasis.

\section{Discussion}

Monoclonal antibody E48 specifically recognises squamous and transitional epithelia and the carcinomas derived from it. Its usefulness in the detection of squamous cell carcinoma has been shown. ${ }^{1822}$ We have shown that E48 can differentiate urothelial carcinomas. Many studies have investigated the usefulness of various antigens as prognostic indicators of biological behaviour of TCCs. ${ }^{347-151722}$ None of them is specific for TCC, and their expression varies strongly with tumour grade. In contrast, E48 specifically recognises squamous and TCCs, without significant loss of reactivity in poorly differentiated carcinomas. Our study shows that it is useful for identifying the urothelial origin of poorly differentiated bladder carcinoma and its metastases.

Previous studies ${ }^{1821}$ have proved that the antigen recognised by E48 is a 22 kilodalton peptide epitope with one or more disulphide bands. Immunoelectron microscopic analysis indicates that the E48 antigen is localised on the cytoplasmic membrane and is largely associated with desmosomes. Knowledge of the function of the E48 antigen is paltry. The localisation of the antigen, the prevention of cell-cell contact formation in the presence of E48 during in vitro culturing of squamous cell carcinoma, and the increased expression of the E48 antigen when cells are grown under culture conditions which promote three-dimensional growth, suggest that the E48 antigen is directly involved in organotopic intercellular adhesions. ${ }^{20}$

The high sensitivity of E48 to TCCs was shown by the reactivity of $91 \%$ of carcinomas investigated in this study. Loss of antibody reactivity in poorly differentiated carcinomas is a well known phenomenon which might be due to maturation or differentiation arrest. For example, the expression of cytokeratin polypeptides has been shown to depend on tumour grading ${ }^{3411}$ and progression. ${ }^{4121323}$ to the fixation method used. In ninety per cent of cases cell membrane bound expression of E48 was most prominent (fig 2), frequently $(36 \%)$ accompanied by staining of the cytoplasm. In $10 \%$ of cases only cytoplasmic expression was found. Background staining 

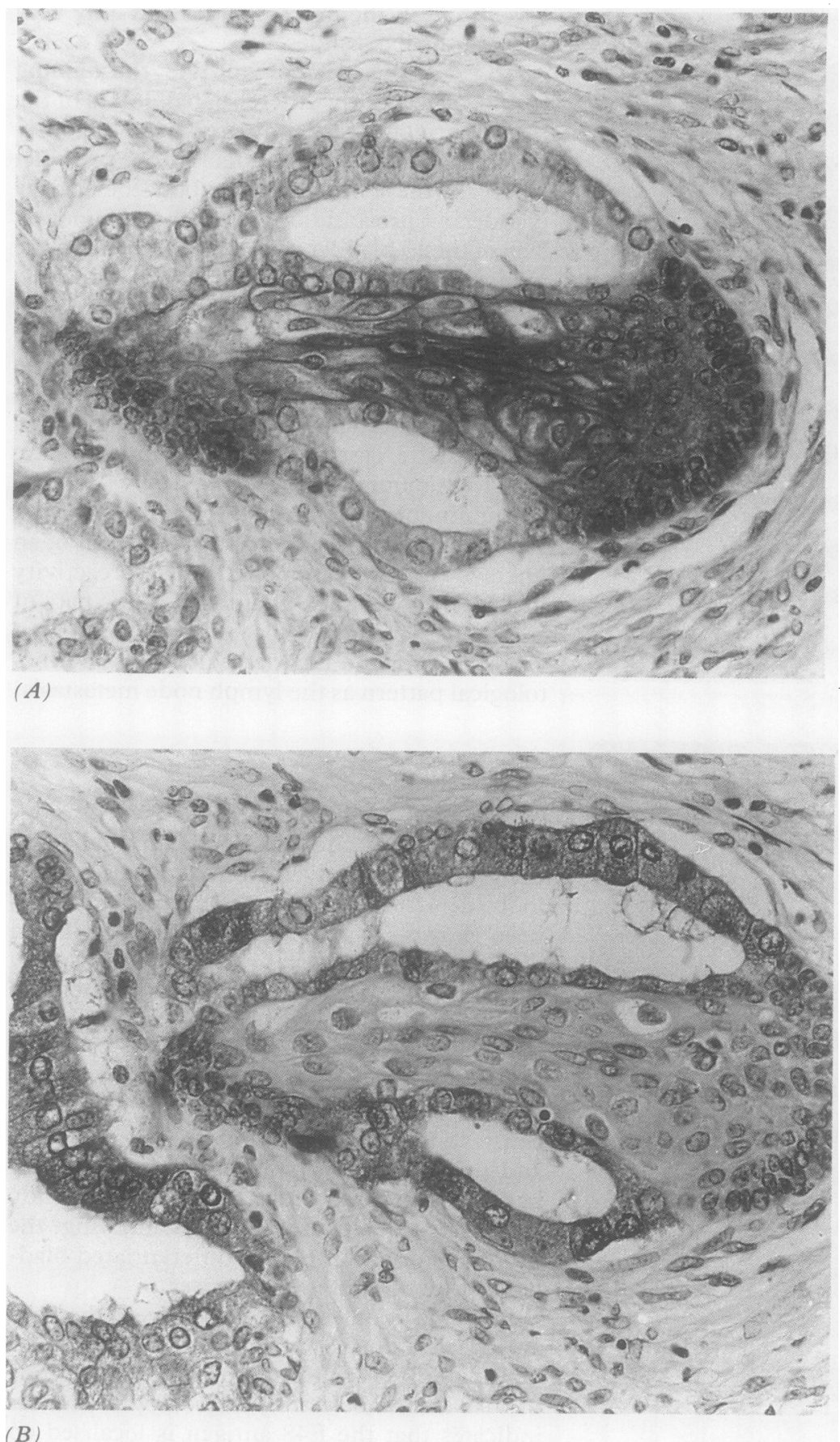

(B)

Figure 3 Squamous metaplasia of the prostate showing positive immunostaining with E48 ( $A)$, but not with prostate specific antigen (B) (formalin fixed).

Although there was a shift from diffuse to focal staining, E48 was still detectable in almost all cases of moderately and poorly differentiated TCCs $(94 \%$ and $83 \%$ respectively), which makes this antibody very appropriate as an identification marker for TCC.

Our findings indicate the importance of adequate tissue preparation for preservation of the epitope. Optimal staining results with E48 are obtained when tissue samples are fresh frozen. Half the tumours which showed reactivity to E48 on fresh frozen tissue samples lost expression of a part of the tumour, and $18 \%$ of the formalin fixed samples became completely negative. Furthermore, in tissue samples obtained at necropsy, the antigen is seldom expressed. Apparently, the epitope recognised by $\mathrm{E} 48$ might be masked or destroyed by fixation, and is susceptible to autolysis. Staining results of the sublimate formalin fixed samples did improve with pretreatment with trypsin by which the epitope might be demasked.

The diagnostic relevance of E48 is shown in the differential diagnosis of high grade TCC compared with poorly differentiated prostatic carcinoma. Histologically, it may be nearly impossible to differentiate between both tumours. Moreover, both malignancies may co-exist and a tumour of one organ can spread into the other. ${ }^{24}$ Discrimination, however, is very important with regard to therapeutic management. The use of PSA or prostatic acid phosphatase (PRAP) allows the prostatic origin of poorly differentiated carcinomas to be identified in about $90 \%$ of cases, ${ }^{25-29}$ although focal reactivity of PRAP has also been reported in $30 \%$ of adenocarcinomas of the bladder. ${ }^{30}$ In cases of uncertain origin, and especially if prostate markers are negative, an additional urothelial marker may help in reaching a final diagnosis. The marker also may also help to differentiate from other adenocarcinomas infiltrating the bladder, such as those derived from the lower intestinal, or female genital tract, which will not be labelled by E48. ${ }^{18}$

Special attention must be paid to squamous or transitional metaplasia of the prostate which occasionally occurs, especially in areas of ischaemic scarring. Positive immunostaining with E48 of such foci within areas of poorly differentiated carcinoma poses a diagnostic problem. The coexistence of ischaemic necrosis and fibrous scar tissue and the lack of anaplasia and invasion ${ }^{28}$ may help to recognise these metaplastic foci and prevent a false diagnosis of TCC.

Another well known diagnostic problem arises when a metastasis occurs from an unknown primary malignancy. Reactivity of such a lesion for E48 led us to consider a metastasis of a primary transitional cell carcinoma, which was confirmed by cystoscopy.

In conclusion, E48 is a sensitive monoclonal antibody that can be used to determine the urothelial origin of poorly differentiated carcinomas in the pelvic region and of metastases of unknown primary tumours. The high specificity for transitional cell and squamous cell epithelium, as well as preserved reactivity after formalin fixation, makes the antibody valuable for diagnostic purposes. Moreover, the accessibility of the epitope on the outer surface in most TCCs and the restricted expression in other tissues of the body suggest that the antibody is a suitable candidate for immunotargeting. In fact, squamous cell carcinoma xenografts in nude mice could be localised successfully with radiolabelled E48. ${ }^{21}$ The antigen recognised by $\mathrm{E} 48$ seems to have a role in cell-cell interaction, but its precise role as a tissue specific antigen has still to be determined.

1 Takahashi N, Takahashi S, Takahashi K, Suzuki T, Funyu $T$. A monoclonal antibody to human transitional cell carcinoma of the bladder: production and characterizaBraesch-Andersen S, Paull S, 


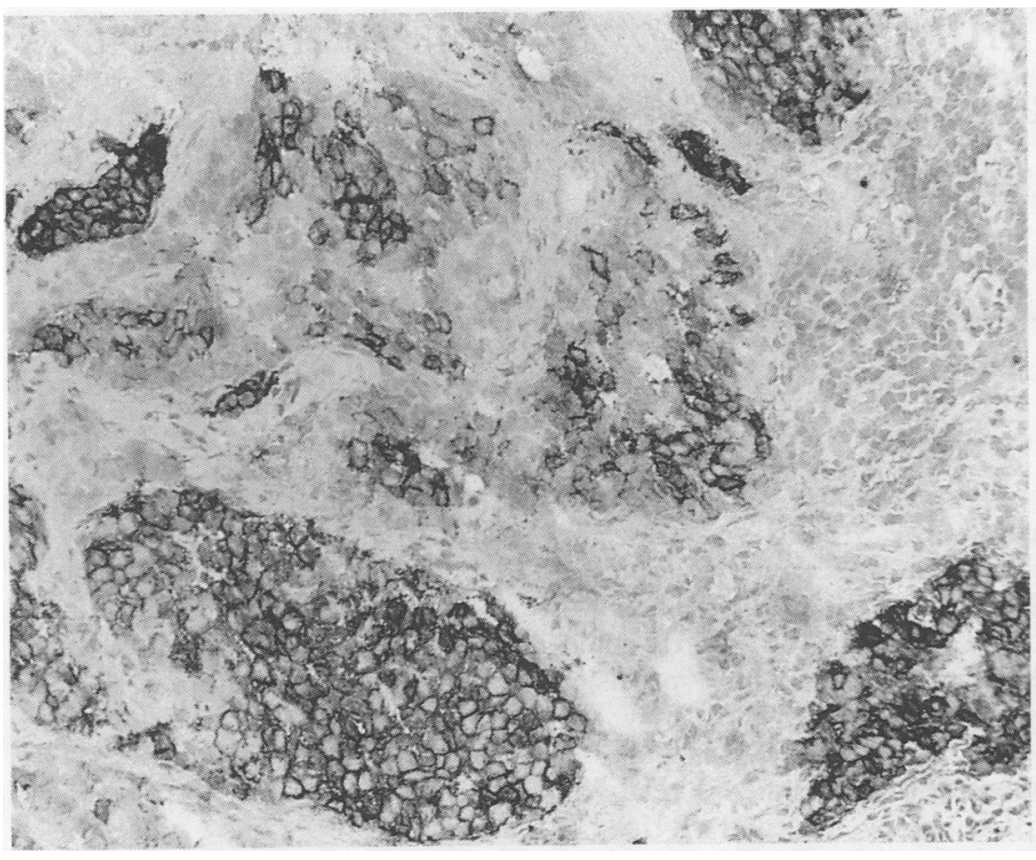

Figure 4 Cervical lymph node containing a metastasis of a poorly differentiated transitional cell carcinoma reacting with E48 (fresh frozen).

tion and characterization of two bladder carcinomaassociated antigens. J Immunol Methods 1986;94:145-51

Asamoto M, Fukushima S, Tatemoto Y, Yamada K, Fukui S, Mori M. Immunohistochemical expression of keratin proteins in urinary bladder carcinoma. Path Res Pract 1989;184:194-201.

4 Achtstatter T, Moll R, Moore B, Franke WW. Cytokeratin polypeptide patterns of different epithelia of the human male urogenital tract: Immunofluorescence and gel electrophoretic studies. J Histochem Cytochem 1985;33: trophore

5 Schaafsma HE, Ramaekers FCS, Muijen van GNP, et al. Distribution of cytokeratin polypeptides in human transitional carcinomas, with special emphasis on changing expression patterns during tumour progression. $A m$ Pathol 1990;136:329-43.

6 Sheibani K, Battifora H, Burke JS, Rappaport H. Leu M1 antigen in human neoplasms. Am J Surg Patho 1986;10:227-36.

7 Dexeus F, Logothetis C, Hossan E, Samuels ML. Carcinoembryonic antigen and beta-human chorionic gonadotropin as serum markers for advanced urothelial malignancies. J Urol 1986;136:403-7.

8 Hall RR. Carcinoembryonic antigen and urological carcinoma: A review after 7 years. Br J Urol 1980;52:166.

9 Swanson PE. Monoclonal antibodies to human milk fat gobulin proteins. In: Wick MR, Siegal GP, eds. Monoclonal antibodies in diagnostic immunohistochemistry. New York: Marcel Dekker, 1988:227.

10 Moll R, Achtstatter T, Becht E, Balcarova-Stander J, Ittensohn M, Franke WW. Cytokeratins in normal and malignant transitional epithelium: maintenance of expression of urothelial differentiation features in transitional cell carcinomas and bladder carcinoma cell culture lines. $\mathrm{Am} J$ Pathol 1988;132:123-44.

11 Moll R, Franke WW, Schiller DL, Geiger B, Krepler R. The catalogue of human cytokeratin polypeptides: patterns of expression of cytokeratin in normal epithelium, tumours, and cultured cells. Cell 1982;31:11-24.

12 Debus E, Moll R, Franke WW, Weber K, Osborn M Immunohistochemical distinction of human carcinomas by cytokeratin typing with monoclonal antibodies. $\mathrm{Am}$ Pathol 1984;114:121-30.

13 Ramaekers F, Huysmans A, Moesker O, Schaart G, Herman C, Vooijs P. Cytokeratin expression during neoplastic progression of human transitional cell carcinomas as detected by a monoclonal and polyclonal antibody. Lab detected by a monoclo

14 Hakomori SI. Bloodgroup glycolipid antigens and their modifications as human cancer antigens. Am J Clin Patho modifications

15 Coon JS, Weinstein RS. Variability in the expression of the $\mathrm{O}(\mathrm{H})$ antigen in human transitional epithelium. $J$ Urol 1981;125:301-6.

16 Springer GF, Desai PR, Murthy MS, et al. Precursors of the bloodgroup $\mathrm{MN}$ antigens as human carcinoma associated antigens. Transfusion 1979;19:233-49.

17 Lloyd KO. Blood group antigens as markers for norma differentiation and malignant change in human tissues. $A m$ J Clin Pathol 1987:87:129-39.

18 Quak JJ, Balm AJM, Dongen GAMS van, et al. A 22kD surface antigen detected by monoclonal antibody E48 is surface antigen detected by monoclonal antibody $\mathrm{E} 48$ is sitional epithelia. Am J Pathol 1990;136:191-7.

19 Spitz M, Spitz L, Thorpe R, Eugui E. Intrasplenic immunization for the production of monoclonal antibodies. Immunol Methods 1984;70:39-43.

20 Schrijvers AHGJ, Gerretsen M, Fritz JM, et al. Evidence for the role of the monoclonal antibody E48 defined antigen in cell-cell adhesion in squamous epithelia and head and neck squamous cell carcinoma. Exp Cell Res 1991;96: 264-9.

21 Quak JJ, Balm AJM, Brakkee JPG, et al Localization and imaging of radiolabelled monoclonal antibody against cquamous cell carcinoma of the head and neck in tumourbearin nude mice. Int J Cancer 1989;44:534-8.

22 Chopin DK, deKernion JB, Rosenthal DL, Fahey JL. Monoclonal antibodies against transitional cell carcinoma Monoclonal antibodies against transition cells in bladder washing. J Urol 1985;134:260-5.

23 Ramaekers FCS, Moesker O, Huysmans A, et al. Intermediate filament proteins in the study of tumour heterogeneity. In: Boland B, Hitchcock J, Brenner MK eds. An in-depth study of tumours of the urinary and respiratory tracts. New York: The New York Academy of Sciences, Inc, 1985:614-34.

24 Koss LG. Tumours of the urinary bladder. In: Atlas of tumour pathology. Washington, DC: Armed Forces Institute of Pathology, 1975

25 Jobsis AC, Vries de GP, Meijer AEFH, Ploem JS. The immunohistochemical detection of prostatic acid phosimmunohistochemical detection of prostatic acid phosphatase: its possibilities and 13:961-73.

26 Nadji M, Tabei SZ, Castro A, et al. Prostatic-specific antigen: An immunohistologic marker for prostatic neoantigen: An immunohistologic mar

27 Feiner HD, Gonzalez R. Carcinoma of the prostate with atypical immunohistochemical features. Am J Surg Pathol 1986;10:765-70.

28 Mostofi FK, Price EB. Tumours of the male genital system In: Atlas of tumour pathology. Washington, DC. Armed Forces Institute of Pathology, 1973.

29 Nadju M, Tabei MDS, Castro A, Chu TM, Morales AR. Prostate origin of tumours. Am J Clin Pathol 1980;73: 735-9.

30 Epstein JI, Kuhajda FP, Lieberman PH. Prostate specific phosphatase immunoreactivity in adenocarcinomas of the urinary bladder. Hum Pathol 1986;17:939-42. 